\title{
THE SIX DIMENSIONAL GAUSS BONNET INTEGRAND
}

\author{
ALFRED GRAY ${ }^{1}$
}

\begin{abstract}
Sufficient conditions are given for a compact 6dimensional Kähler manifold with nonnegative (nonpositive) curvature to have nonnegative (nonpositive) Euler characteristic.
\end{abstract}

1. Introduction. One of the most important unresolved conjectures in differential geometry is the following:

(*) Let $M$ be a compact oriented Riemannian manifold of even dimension $n=2 m$. If all the sectional curvatures of $M$ are nonnegative then the Euler characteristic $\chi(M) \geqq 0$. If all the sectional curvatures of $M$ are nonpositive then $(-1)^{m} \chi(M) \geqq 0$.

This conjecture is easy to prove if $\operatorname{dim} M=2$ and has also been proved in the 4-dimensional case by J. Milnor (see [2] and [3]). In fact if $\operatorname{dim} M=$ 4 , much weaker assumptions imply $\chi(M) \geqq 0$. It suffices to assume that $M$ has positive Ricci curvature or that $M$ is Kählerian and the holomorphic sectional curvatures of $M$ all have the same sign (see [2]).

The main line of attack on the conjecture $(*)$ has been to make use of the Gauss Bonnet formula. This involves estimating the Gauss Bonnet integrand in terms of sectional curvature. In the 4-dimensional case the Gauss Bonnet integrand is a quadratic polynomial in 20 variables, but it is manageable. However, in the general 6-dimensional case the Gauss Bonnet integrand is a cubic polynomial in 105 variables, and it would be very tedious just to write it out. See [2] for a discussion of some of the problems involved in estimating it. It is expedient, therefore, to look at classes of 6-dimensional Riemannian manifolds for which the Gauss Bonnet integrand is less formidable.

Let $\mathscr{R}$ be the vector space of all tensors which satisfy all the identities of the curvature tensor of a 6-dimensional Riemannian manifold. Then

Received by the editors March 24, 1972.

AMS (MOS) subject classifications (1970). Primary 53C30, 53C55, 53C65; Secondary 58A10, 57D20.

Key words and phrases. Gauss Bonnet, Euler characteristic, Kähler manifold, Einstein manifold, sectional curvature, Ricci curvature.

${ }^{1}$ This work was partially supported by National Science Foundation grant 27451.

(c) American Mathematical Society 1973 
$\mathscr{R}$ has dimension 105 and has subspaces $\mathscr{R}_{\mathrm{e}}, \mathscr{R}_{\mathrm{k}}$, and $\mathscr{R}_{\mathrm{ke}}$ where

$$
\begin{aligned}
\mathscr{R}_{\mathrm{e}} & =\{R \in \mathscr{R} \mid R \text { is Einstein }\}, \\
\mathscr{R}_{\mathrm{k}} & =\{R \in \mathscr{R} \mid R \text { is Kählerian }\}, \\
\mathscr{R}_{\mathrm{ke}} & =\mathscr{R}_{\mathrm{e}} \cap \mathscr{R}_{\mathrm{k}} .
\end{aligned}
$$

Here $\operatorname{dim} \mathscr{R}_{\mathrm{e}}=85, \operatorname{dim} \mathscr{R}_{\mathrm{k}}=36$, and $\operatorname{dim} \mathscr{R}_{\mathrm{ke}}=28$. The space $\mathscr{R}_{\mathrm{e}}$ is still quite large; however, $\mathscr{R}_{\mathrm{k}}$ and $\mathscr{R}_{\mathrm{ke}}$ are small enough for us to say something about the Gauss Bonnet integrand for curvature operators in these spaces.

In $\S 2$ we write down the Gauss Bonnet integrand for 6-dimensional Kähler manifolds. Then in $\S 3$ we generalize a theorem of Bishop and Goldberg [2] in several ways. In each case we prove that for a curvature tensor $R \in \mathscr{R}_{\mathrm{ke}}$ with nonnegative (nonpositive) sectional curvature the Gauss Bonnet integrand is nonnegative (nonpositive) provided that certain components of the curvature tensor vanish.

At this point I do not know whether the conjecture $(*)$ is true for compact 6-dimensional Kähler manifolds, or even Einstein Kähler manifolds. The evidence so far obtained is the following.

Positive evidence. For all the cases computed so far it turns out that (*) is true. It is shown in Theorem 2 below that $(*)$ is true provided that at each point the curvature tensor lies in a certain 14-dimensional subspace of the 28-dimensional space $\mathscr{R}_{\mathrm{ke}}$. Thus $(*)$ is not too far from being settled for compact 6-dimensional Einstein Kähler manifolds.

Negative evidence. There is a great deal of difference between the 4- and 6-dimensional cases. For example nonnegative homomorphic sectional curvature implies that the Gauss Bonnet integrand is nonnegative in the 4-dimensional case. Bishop and Goldberg [2] have given an example to show that this is no longer true for 6-dimensional Kähler manifolds.

It is of course possible that the Gauss Bonnet integrand is negative for some curvature operator with nonnegative sectional curvature but (*) is still true. For example, it is not difficult to construct a 4-dimensional curvature tensor with positive Ricci curvature and negative Gauss Bonnet integrand. However, a compact 4-dimensional Riemannian manifold with positive Ricci curvature has positive Euler characteristic.

In this connection the following result of F. Rideau [5] is relevant.

THEOREM. Let $M$ be a compact Kähler manifold with positive holomorphic bisectional curvature. If $h^{p, q}(M)$ denotes the dimension of the space of harmonic $(p, q)$-forms, then $h^{p, 1}(M)=h^{1, p}(M)=0$ for $p>1$.

COROLlary. If $M$ is a compact 6-dimensional Kähler manifold with positive holomorphic bisectional curvature then $\chi(M)>0$. 
Proof. It is well known (see for example [7]) that for a compact Kähler manifold with positive Ricci curvature we always have $h^{p, 0}(M)=$ $h^{0, p}(M)=0$ for $p>0$. This fact together with Rideau's result and Poincaré duality imply that all the odd Betti numbers of $M$ are zero. Hence $\chi(M)>0$.

Positive sectional curvature imply positive holomorphic bisectional curvature. On the other hand in the example of Bishop and Goldberg cited above the curvature operator has positive holomorphic bisectional curvature but negative Gauss Bonnet integrand.

2. The Gauss Bonnet integrand of a 6-dimensional Kähler manifold. In dimension 6 the Gauss Bonnet formula is

$$
\chi(M)=\frac{1}{8 \pi^{2}} \int_{M} \Omega_{123456} .
$$

Here $\Omega_{i j}$ is the curvature form of $M$. Let

$$
\Omega_{i j k l}=\Omega_{i j} \wedge \Omega_{k l}-\Omega_{i k} \wedge \Omega_{j l}+\Omega_{i l} \wedge \Omega_{j k} .
$$

Then $\Omega_{123456}$ is defined by

$$
\begin{aligned}
\Omega_{123456}= & \Omega_{12} \wedge \Omega_{3456}-\Omega_{13} \wedge \Omega_{2456}+\Omega_{14} \wedge \Omega_{2356} \\
& -\Omega_{15} \wedge \Omega_{2346}+\Omega_{16} \wedge \Omega_{2345} .
\end{aligned}
$$

Let $\left\{e_{1}, \cdots, e_{6}\right\}$ be an orthonormal basis of a tangent space $M_{m}$ and let $R$ denote the curvature operator of $M$. Then $R$ is related to the $\Omega_{i j}$ 's by the formulas

We also put

$$
\left\langle R_{e_{i e j}} e_{k}, e_{l}\right\rangle=R_{i j k l}=\Omega_{i j}\left(e_{k}, e_{l}\right)
$$

$$
R_{i j k l p q r s}=\Omega_{i j k l}\left(e_{p}, e_{q}, e_{r}, e_{s}\right) .
$$

It is well known that the components of the curvature tensor $R_{i j k l}$ satisfy the Bianchi identity

$$
R_{i j k l}+R_{k i j l}+R_{j k i l}=0 .
$$

Furthermore in [4] and [6] it is shown that another "Bianchi" identity

$$
R_{i j k l p q r s}+R_{\text {pijklqrs }}+R_{l p i j k q r s}+R_{k l p i j q r s}+R_{j k l p i q r s}=0
$$

is satisfied.

The 2-, 4-, and 6-dimensional sectional curvatures are given by the formulas

$$
\begin{aligned}
K_{i j} & =R_{i j i j}=\Omega_{i j}\left(e_{i}, e_{j}\right), \\
K_{i j k l} & =R_{i j k l i j k l}=\Omega_{i j k l}\left(e_{i}, e_{j}, e_{k}, e_{l}\right), \\
K_{123456} & =\Omega_{123456}\left(e_{1}, e_{2}, e_{3}, e_{4}, e_{5}, e_{6}\right) .
\end{aligned}
$$


We now specialize to the case of a 6-dimensional Kähler manifold $M$ and take an orthonormal basis of $M_{m}$ of the form $\left\{e_{1}, J e_{1}, e_{2}, J e_{2}, e_{3}, J e_{3}\right\}$ where $J$ denotes the almost complex structure of $M$. We write $e_{i}{ }^{*}=J e_{i}$ for $i=1,2,3$.

It is well known that for a Kähler manifold we have

$$
R_{i j k^{*} l^{*}}=R_{i j k l},
$$

and that (1) and (3) imply

$$
R_{i i^{*} j j^{*}}=K_{i j}+K_{i j^{*}} .
$$

It will be useful to have similar formulas for the $R_{i j k l p a r s}$. It is not hard to see that

$$
R_{i j k l p^{*} q^{*} r^{*} s^{*}}=R_{i j l l p q r s}
$$

Then from (2) and (5) we obtain

$$
R_{i i^{*} j j^{*} i i^{*} k k^{*}}=K_{i i}{ }^{*} j k+K_{i i^{*} j k^{*}} .
$$

Using these formulas we derive a formula for the Gauss Bonnet integrand of a 6-dimensional Kähler manifold. First we observe that

$$
\begin{aligned}
& 3 \Omega_{11^{*} 22^{*} 33^{*}=} \Omega_{11^{*}} \wedge \Omega_{22^{*} 33^{*}}+\Omega_{22^{*}} \wedge \Omega_{111^{*} 33^{*}}+\Omega_{33^{*}} \wedge \Omega_{11^{*} 22^{*}} \\
& -2\left\{\Omega_{12} \wedge \Omega_{1233^{*}}+\Omega_{12^{*}} \wedge \Omega_{12^{*} 33^{*}}+\Omega_{13} \wedge \Omega_{1322^{*}}\right. \\
& \left.+\Omega_{13^{*}} \wedge \Omega_{13^{*} 22^{*}}+\Omega_{23} \wedge \Omega_{2311^{*}}+\Omega_{23^{*}} \wedge \Omega_{23^{*} 11^{*}}\right\}
\end{aligned}
$$

We evaluate each of the differential forms on the right-hand side of (7) on $\left\{e_{1}, e_{1^{*}}, e_{2}, e_{2^{*}}, e_{3}, e_{3^{*}}\right\}$. Adding the results and making use of (4) and (6) we obtain

$$
\begin{aligned}
& 3 K_{11^{*} 22^{*} 33^{*}}=3 \Omega_{11^{*} 22^{*} 33^{*}}\left(e_{1}, e_{1^{*}}, e_{2}, e_{2^{*}}, e_{3}, e_{3^{*}}\right) \\
& =K_{11}{ }^{*} K_{22^{*} 3^{*}}+K_{22^{*}} K_{11^{*} 3^{*}}+K_{33^{*}} K_{11^{*} 22^{*}} \\
& +2\left\{\left(3 K_{12}+K_{12^{*}}\right) K_{33^{*} 12}+\left(K_{12}+3 K_{12^{*}}\right) K_{33^{*} 12^{*}}\right. \\
& +\left(3 K_{13}+K_{13}{ }^{*}\right) K_{22}{ }^{*} 13+\left(K_{13}+3 K_{13}\right) K_{22^{*} 13^{*}} \\
& +\left(3 K_{23}+K_{23^{*}}\right) K_{11^{*}}{ }^{23}+\left(K_{23}+3 K_{23^{*}}\right) K_{\left.11^{*}{ }^{*} 3^{*}\right\}} \\
& +8\left\{R_{1212^{*}} R_{33^{*} 1233^{*} 12^{*}}+R_{1313^{*}} R_{22^{*} 1322^{*} 13^{*}}\right. \\
& \left.+R_{2323^{*}} R_{11^{*}}{ }^{2311^{*}}{ }^{*} 3^{*}\right\}
\end{aligned}
$$

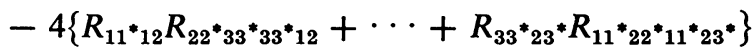

$$
\begin{aligned}
& +4\left\{\left(3 R_{1213}+R_{12^{*} 13^{*}}\right) R_{33^{*} 1222^{*} 13}+\cdots\right. \\
& \left.+\left(R_{1323}+3 R_{1^{*} 32^{*} 3}\right) R_{22^{*} 1311^{*} 23}\right\} \text {. }
\end{aligned}
$$


This is the Gauss Bonnet integrand for a 6-dimensional Kähler manifold.

3. Sufficient conditions that the Gauss Bonnet integrand be nonnegative or nonpositive. We now use $(8)$ to prove $(*)$ in some special cases.

THEOREM 1. Let $M$ be a compact 6-dimensional Kähler manifold such that for each $m \in M$ the tangent space $M_{m}$ has an orthonormal basis $\left\{e_{1}, J e_{1}, e_{2}, J e_{2}, e_{3}, J e_{3}\right\}$ with the following property: the eigenvectors of $R_{e_{i} J e_{i}} J$ are $e_{1}, J e_{1}, e_{2}, J e_{2}, e_{3}, J e_{3}$ (where $R$ denotes the curvature operator of $M)$. If $M$ has nonnegative (nonpositive) sectional curvature, then $\chi(M) \geqq 0(\chi(M) \leqq 0)$.

The hypotheses of the theorem arise from the following considerations. Choose $e_{1}$ so that $K_{11^{*}}$ is a maximum holomorphic sectional curvature. Then an orthonormal basis which diagonalizes the symmetric operator $R_{e_{1} J e_{1}} J$ is of the form $\left\{e_{1}, J e_{1}, e_{2}, J e_{2}, e_{3}, J e_{3}\right\}$. The hypotheses of the theorem imply that this basis also diagonalizes $R_{e_{2} J e_{2}} J$ and $R_{e_{3} J e_{3}} J$.

In [2] Bishop and Goldberg prove that if all the components of the curvature tensor other than the sectional curvatures vanish, then $K_{11 * 22^{*} 3 *} \geqq 0$. Whereas Bishop and Goldberg prove $(*)$ for a certain 9dimensional subspace of $\mathscr{R}_{\mathrm{k}}$, Theorem 1 proves $(*)$ for a 18 -dimensional subspace of the 36 -dimensional space $\mathscr{R}_{\mathrm{k}}$.

As a consequence of Theorem 1 we shall obtain the following theorem in which the curvature conditions are more geometric.

THEOREM 2. Let $M$ be a compact 6-dimensional Einstein Kähler manifold. Assume that for each $m \in M$ the holomorphic sectional curvature assumes critical values on a triple of mutually orthogonal holomorphic sections in $M_{m}$. If $M$ has nonnegative (nonpositive) sectional curvatures then $\chi(M) \geqq 0(\chi(M) \leqq 0)$.

Proof OF THEOREM 1. We have

$$
R_{11}{ }^{* 12}=\cdots=R_{33}{ }^{*} 3^{*}=0,
$$

and

$$
R_{11{ }^{*} 23}=\cdots=R_{33^{*} 12^{*}}=0 .
$$

From (10) it follows that

$$
R_{i j i k}=-R_{i j^{*} i k^{*}} \text { for } i, j, k \text {, all different. }
$$

Furthermore a calculation shows that

$$
\begin{aligned}
R_{i i^{*} j k j j^{*} i k} & =-R_{i i^{*} j^{*} k j j^{*} i^{*} k} \\
& =2\left(K_{i j}+K_{i j^{*}}\right) R_{i k j k}-2 R_{i j i k} R_{i j k j}+2 R_{i j^{*} i k} R_{i^{*} j k j} .
\end{aligned}
$$


From (8), (9), (10), (11), and (12) we obtain

$$
\begin{aligned}
& 3 K_{11}{ }^{*}{ }^{2}{ }^{*} 33^{*}=K_{11^{*}} K_{22}{ }^{*} 33^{*}+K_{22^{*}} K_{11^{*} 33^{*}}+K_{33^{*}} K_{11^{*}}{ }_{22^{*}} \\
& +\left(6 K_{12}+2 K_{12^{*}}\right) K_{33^{*} 12}+\cdots+\left(2 K_{23}+6 K_{23}\right) K_{11^{*} 23^{*}} \\
& +8\left(R_{1212^{*}} R_{33^{*} 1233^{*} 12^{*}}+R_{1313^{*}} R_{22^{*} 1322^{*} 13^{*}}\right. \\
& +R_{2323^{*}} R_{\left.11^{*}{ }^{* 311^{*}}{ }^{*} 3^{*}\right)} \\
& +32\left\{\left(K_{12}+K_{12}\right)\left(R_{1323}^{2}+R_{1^{*} 323}^{2}\right)\right. \\
& +\left(K_{13}+K_{13} *\right)\left(R_{1232}^{2}+R_{1 * 232}^{2}\right) \\
& \left.+\left(K_{23}+K_{23^{*}}\right)\left(R_{1213}^{2}+R_{12^{*} 13}^{2}\right)\right\} \\
& +96\left\{-R_{1213} R_{1232} R_{1323}+R_{12}{ }^{*}{ }_{13} R_{1}{ }^{*}{ }_{232} R_{1323}\right.
\end{aligned}
$$

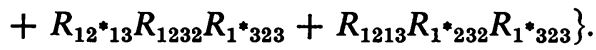

Since $M$ has nonnegative or nonpositive sectional curvatures, $\left|R_{i j i k}\right| \leqq$ $\left|K_{i j} K_{i k}\right|^{1 / 2}$ (see [1]). Hence if $M$ has nonnegative sectional curvature

$$
\begin{aligned}
& K_{12} R_{1323}^{2}-2 R_{1213} R_{1323} R_{1232}+K_{13} R_{1232}^{2} \geqq 0, \\
& K_{12} R_{1323}^{2}-2 R_{1213} R_{1323} R_{1232}+K_{23} R_{1213}^{2} \geqq 0, \\
& K_{13} R_{1232}^{2}-2 R_{1213} R_{1323} R_{1232}+K_{23} R_{1213}^{2} \geqq 0, \quad \text { etc. }
\end{aligned}
$$

From (14) we obtain

(15) $32\left(K_{12} R_{1323}^{2}+K_{13} R_{1232}^{2}+K_{23} R_{1213}^{2}-3 R_{1213} R_{1323} R_{1232}\right) \geqq 0$, etc.

The same argument that proves $\left|R_{i j i k}\right| \leqq\left(K_{i j} K_{i k}\right)^{1 / 2}$ shows also that $\left|R_{33^{*} 1233^{*} 12^{*}}\right| \leqq\left(K_{33^{*} 12} K_{33^{*} 2^{*}}\right)^{1 / 2}$. Hence

$$
\begin{aligned}
8\left|R_{1212}{ }^{*} R_{33^{*} 1233^{*} 12^{*}}\right| & \leqq 8\left|K_{12} K_{33^{*} 12} K_{12}{ }^{*} K_{33^{*} 12^{*}}\right|^{1 / 2} \\
& \leqq 4\left(\left|K_{12}\right|\left|K_{33^{*} 12}\right|+\left|K_{12}{ }^{*}\right|\left|K_{33^{*} 12^{*}}\right|\right), \text { etc. }
\end{aligned}
$$

Therefore from (13), (15), and (16) we obtain

$$
\begin{aligned}
3 K_{11^{*} 22^{*} 33^{*} \geqq} & K_{11^{*}} K_{22^{*} 33^{*}}+K_{22^{*}} K_{11^{*} 33^{*}}+K_{33^{*}} K_{11}{ }^{*} 2^{*} \\
& +2\left(K_{12}+K_{12}\right)\left(K_{33^{*} 12}+K_{33^{*} 1^{*}}\right) \\
& +2\left(K_{13}+K_{13^{*}}\right)\left(K_{22^{*} 13}+K_{22^{*} 13^{*}}\right) \\
& +2\left(K_{23}+K_{23^{*}}\right)\left(K_{11^{*}}{ }^{*}+K_{11^{*} 23^{*}}\right) \\
\geqq & 0,
\end{aligned}
$$

provided that $M$ has nonnegative sectional curvature. If $M$ has nonpositive sectional curvature a similar argument shows that (17) holds with the inequalities reversed. Q.E.D. 
Proof of TheOrem 2. Since $M$ is an Einstein Kähler manifold we have

$$
\begin{aligned}
0 & =R_{i i^{*} i j}+R_{j^{*} i^{*} j^{*} j}+R_{k i^{*} k j}+R_{k^{*} i^{*} k^{*} j} \\
& =R_{i i^{*} i j}+R_{j j^{*} i j}+R_{k k^{*} i j} \quad(i, j, k \neq) .
\end{aligned}
$$

The hypotheses of Theorem 2 imply that $\left\{e_{1}, e_{1^{*}}, e_{2}, e_{2^{*}}, e_{3}, e_{3^{*}}\right\}$ can be chosen so that $K_{11^{*}}, K_{22}$, and $K_{33^{*}}$ are critical values of the holomorphic sectional curvature; then $R_{i i^{*} i j}=R_{i i^{*} i j^{*}}=0$ for all $i$ and $j$. From (18) it follows that $R_{i i}{ }^{*} j k=R_{i i^{*} j k^{*}}=0$ for $j \neq k$. Thus the hypotheses of Theorem 1 are fulfilled. Q.E.D.

\section{REFERENCES}

1. M. Berger, Sur quelques variétés riemanniennes suffisamment pincées, Bull. Soc. Math. France 88 (1960), 57-71. MR 24 \#A3606.

2. R. L. Bishop and S. I. Goldberg, Some implications of the generalized GaussBonnet theorem, Trans. Amer. Math. Soc. 112 (1964), 508-535. MR 29 \#574.

3. S. S. Chern, On curvature and characteristic classes of a Riemann manifold, Abh. Math. Sem. Univ. Hamburg 20 (1956), 117-126. MR 17, 783.

4. A. Gray, Some relations between curvature and characteristic classes, Math. Ann. 184 (1969/70), 257-267. MR 41 \#6105.

5. F. Rideau, Un théorème sur la courbure holomorphe bissectionelle, C. R. Acad. Sci. Paris Sér. A-B 270 (1970), A887-A889. MR 41 \#44456.

6. J. A. Thorpe, Some remarks on the Gauss-Bonnet integral, J. Math. Mech. 18 (1969), 779-786. MR 41 \#963.

7. K. Yano and S. Bochner, Curvature and Betti numbers, Ann. of Math. Studies, no. 32, Princeton Univ. Press, Princeton, N.J.

Department of Mathematics, The University of Maryland, College Park, MARYLAND 20742 